Return to the Manage Active Submissions page at http://spie.org/app/submissions/tasks.aspx and approve or disapprove this submission. Your manuscript will not be published without this approval. Please contact author_help@spie.org with any questions or concerns.

\title{
Side-pumping Nd:YAG solar laser by six Fresnel lenses
}

\author{
G. Tomás, D. Liang* and J. Almeida \\ CEFITEC, Departamento de Física, FCT, Universidade Nova de Lisboa, 2829-516, \\ Campus de Caparica, Portugal \\ g.tomas@campus.fct.unl.pt, ${ }^{*} \mathrm{dl} @$ fct.unl.pt, jla@campus.fct.unl.pt
}

\begin{abstract}
To obtain a good compromise between collection efficiency and brightness figure of merit of solar-pumped lasers, a new side-pumping scheme is proposed. Firstly the solar radiations are collected and concentrated by six $700 \mathrm{~mm}$ diameter Fresnel lenses. The concentrated solar radiations are subsequently reflected by six plane folding mirrors with $95 \%$ reflectivity, into a common focal spot. This allows the concentration of $1740 \mathrm{~W}$ solar power with about $6.4 \mathrm{~W} / \mathrm{mm}^{2}$ peak solar flux. A secondary concentrator is composed of six aspheric fused silica lenses, positioned around a $40 \mathrm{~mm}$ radius fused silica sphere, compressing all the concentrated solar radiation from the six Fresnel lenses into an $8 \mathrm{~mm}$ diameter by $9 \mathrm{~mm}$ length Nd:YAG single-crystal rod. By positioning the spherical concentrator slightly above the aspherical lenses, a more uniform absorption profile is achieved. Mechanical support with a water cooling system ensures an efficient cooling to the laser medium. Optimal laser parameters are found through $\mathrm{ZEMAX}^{\mathrm{TM}}$ and $\mathrm{LASCAD}^{\mathrm{TM}}$ numerical analysis software. Only $16 \%$ of the solar power is absorbed by Nd:YAG medium. Solar laser power of $42.6 \mathrm{~W}$ is numerically calculated, reaching a collection efficiency of $18.5 \mathrm{~W} / \mathrm{m}^{2}$. For a $400 \mathrm{~mm}$ plane-concave resonance cavity with $-5 \mathrm{~m}$ radius of curvature, $\mathrm{M}_{\mathrm{x}}^{2}=\mathrm{M}_{\mathrm{y}}^{2}=22$ beam quality factors are numerically predicted. A near uniform pump absorption profile can be achieved by increasing the number of Fresnel lens and folding mirrors.
\end{abstract}

Keywords: solar laser, Fresnel lenses, Nd:YAG, collection efficiency, brightness

\section{INTRODUCTION}

The first solid-state sun-pumped laser has been reported by Young in 1966 [1]. Since then, solar lasers have gained an ever increasing importance. They are necessary in remote locations where other forms of energy are scarce and sunlight is abundant. Compared to electrically powered lasers, solar lasers are much simpler and more reliable due to the complete elimination of the electrical power generation and conditioning equipments. This technology has a large potential for many applications, e.g. high-temperature materials processing, renewable magnesium-hydrogen energy cycle, free space laser communications, space to earth power transmission, and so on. Thus, highly efficient solarpumped lasers with excellent beam quality become essential to the success of these applications.

Since the sunlight does not provide enough flux to produce laser, additional focusing systems are required to collect and concentrate the solar radiation in a focal zone. Parabolic mirrors have been exploited to achieve tight focusing of incoming solar radiation [2-5]. $13.9 \mathrm{~W} / \mathrm{m}^{2}$ collection efficiency has been achieved at the PROMES-CNRS (Odeillo, France) in 2012, by pumping a Nd:YAG single-crystal rod through a heliostat - parabolic mirror system [6]. This value is, to the best of our knowledge, the highest solar laser collection efficiency achieved with parabolic mirrors. However, the recent interests in Fresnel lenses, as a much more available, cheaper alternative for solar energy concentration, [7-11] have lead to a significant increase in solar laser collection efficiencies. $19.3 \mathrm{~W} / \mathrm{m}^{2}$ has been reported in 2011 [10] by utilizing an economical Fresnel lens and the most-widely used Nd:YAG single-crystal rod. The recent Nd:YAG solar laser pumped through a large Fresnel lens and a liquid light-guide lens has produced $30.0 \mathrm{~W} / \mathrm{m}^{2}$ record collection efficiency in 2012 [11]. Nevertheless, very large $\mathrm{M}_{\mathrm{x}}{ }^{2}=\mathrm{M}_{\mathrm{y}}{ }^{2}=137$ beam quality factors have also been reported [11], resulting in the brightness figure of merit of only $0.0064 \mathrm{~W}$. Laser beam brightness is defined as the laser power divided by the product of the beam spot area and its solid angle divergence. This product is proportional to the square of beam quality factor $\mathrm{M}^{2}$. Brightness figure of merit $\mathrm{B}$ is then defined [5] as the ratio between laser power and the product of $\mathrm{M}^{2}$ factors.

RIA100 - 254 V. 1 (p.1 of 7) / Color: No / Format: A4 / Date: 5/31/2013 2:46:44 AM

SPIE USE: DB Check, Prod Check, Notes: 
Return to the Manage Active Submissions page at http://spie.org/app/submissions/tasks.aspx and approve or disapprove this submission. Your manuscript will not be published without this approval. Please contact author_help@spie.org with any questions or concerns.

Despite the strong desire to achieve high collection efficiency, more attention should also be paid to the laser beam quality in order to attain tight focusing solar laser beam. To achieve a good compromise between collection efficiency and laser beam quality, an alternative Fresnel lenses - plane mirrors solar pumping approach is put forward in this paper. Solar energy collection and concentration is achieved through six Fresnel lenses with $700 \mathrm{~mm}$ diameter, followed by six plane folding mirrors, which reflect the concentrated solar light into a common focal spot. The highly concentrated solar radiation is then compressed to the lateral surface of the Nd:YAG single-crystal rod through the secondary concentrator. It is constituted by the fused silica sphere, within which the laser rod is installed, and the six fused silica aspherical lenses, positioned around the sphere. The water cooling system ensures an efficient cooling to the laser medium.

Although the most efficient laser systems have end-pumping approaches [10,11], side-pumping configurations are very suitable for laser power scaling. They allow a more uniform pump absorption profile along the laser medium and an even absorbed pump power distribution within it. The associated thermal loading problems can hence be reduced and the brightness figure of merit can be several times higher than that of end-pumping approaches.

Optimum pumping conditions and solar laser beam parameters are found through ZEMAX $^{\mathrm{TM}}$ and LASCAD ${ }^{\mathrm{TM}}$ numerical analysis, respectively. To attain a more efficient side-pumping to the laser rod, the center of the spherical concentrator is placed above the aspherical lenses, which are slightly rotated to redirect the pump light towards the Nd:YAG crystal. Maximum solar laser power of $42.6 \mathrm{~W}$ is expected for the $8 \mathrm{~mm}$ diameter by $9 \mathrm{~mm}$ length Nd:YAG single-crystal rod. Significant collection efficiency of $18.5 \mathrm{~W} / \mathrm{m}^{2}$ is numerically calculated. The beam quality factors $\mathrm{M}_{\mathrm{x}}{ }^{2}=\mathrm{M}_{\mathrm{y}}{ }^{2}=22$ are predicted in this case, resulting in the brightness figure of merit of $0.09 \mathrm{~W}$. This value is 14 times more than that of the most powerful Nd:YAG solar laser [11].

\section{SIX FRESNEL LENSES APPROACH FOR SIDE-PUMPING Nd:YAG SOLAR LASER}

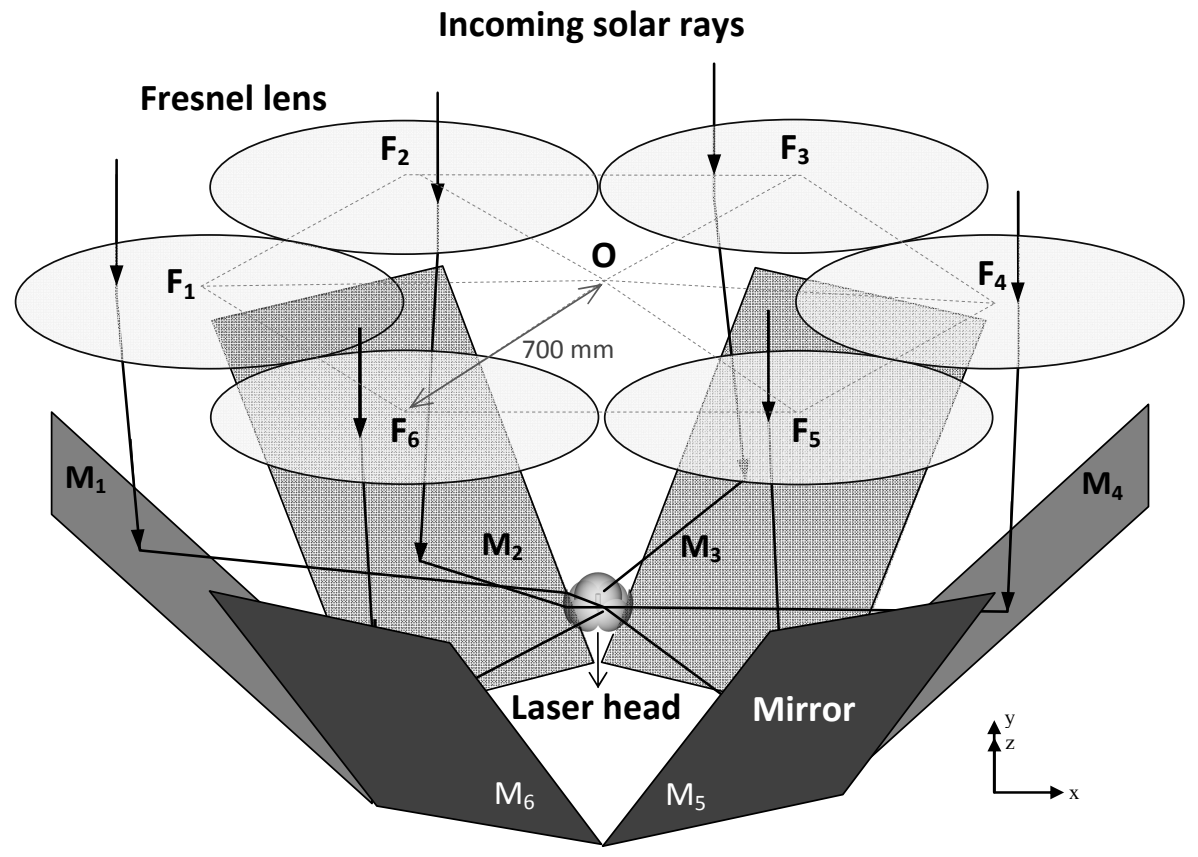

Fig. 1 - Design of the solar laser scheme with 6 Fresnel lenses - 6 plane mirrors. 
Return to the Manage Active Submissions page at http://spie.org/app/submissions/tasks.aspx and approve or disapprove this submission. Your manuscript will not be published without this approval. Please contact author_help@spie.org with any questions or concerns.

\subsection{Solar energy collection and concentration}

The solar-pumping scheme in Fig. 1 is constituted by 6 pairs of circular Fresnel lenses $\left(F_{1}-F_{6}\right)$, which both collect and concentrate the incoming solar radiation, and 6 pairs of plane folding mirrors $\left(\mathrm{M}_{1}-\mathrm{M}_{6}\right)$ that reflect the concentrated solar radiation towards the laser head. The Fresnel lenses have $700 \mathrm{~mm}$ diameter and about $1.3 \mathrm{~m}$ focal length. Each one is positioned at one of the vertices of a virtual hexagon, which are distanced $700 \mathrm{~mm}$ away from their common center $\mathrm{O}$, as shown in Fig. 1. Each folding plane mirror is positioned $575 \mathrm{~mm}$ below its respective Fresnel lens, with a $45^{\circ}$ inclination angle in relation to their common optical axis, as observed in Fig. 2. Solar tracking can be achieved by mounting the whole laser system onto a two axis-heliostat that follows the Sun continuously in direct tracking mode. The total solar energy collection area of $2.3 \mathrm{~m}^{2}$ is considered for the proposed scheme.

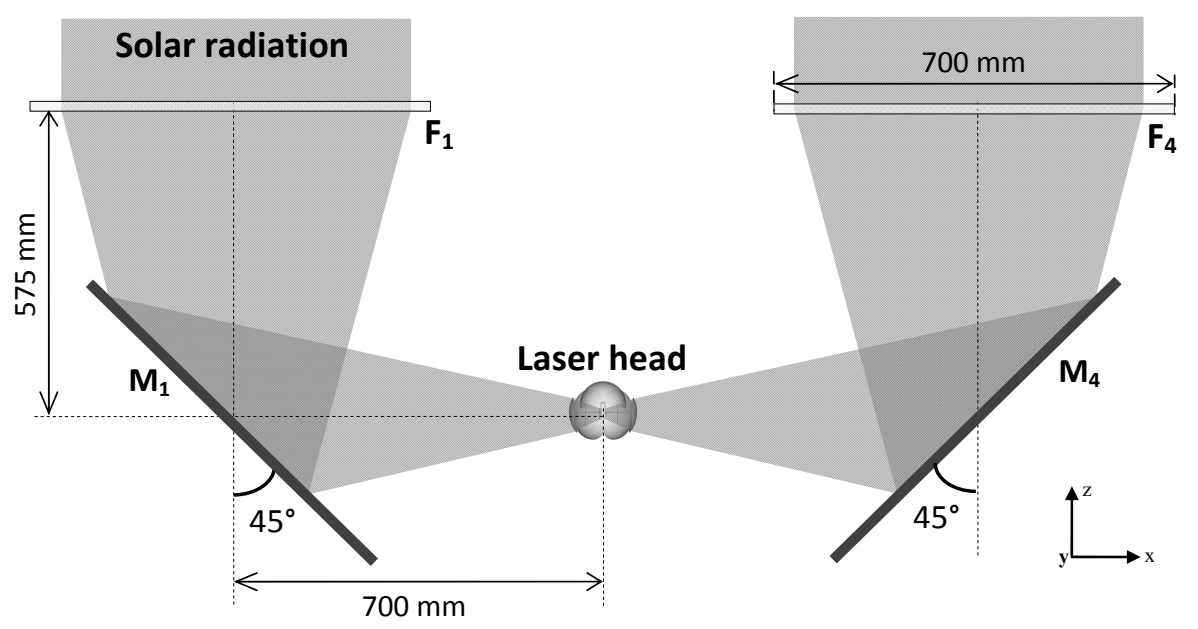

Fig. 2 - Cross-sectional side-view of the solar light collection and concentration system

The Fresnel lenses are made of Polymethyl Methacrylate (PMMA) material, which is transparent at visible and near infrared (NIR) wavelengths, and cuts undesirable solar radiation beyond $2200 \mathrm{~nm}$ and below $350 \mathrm{~nm}$. An averaged transmission efficiency of $84 \%$ is numerically calculated for each Fresnel lens. Considering that each plane mirror reflects $95 \%$ of the useful solar radiation, for $950 \mathrm{~W} / \mathrm{m}^{2}$ terrestrial solar flux, about $1740 \mathrm{~W}$ solar power with about $6.4 \mathrm{~W} / \mathrm{mm}^{2}$ peak flux can be achieved in the focal zone.

\subsection{The solar laser head}

The highly concentrated solar radiation from the 6 Fresnel lenses - 6 plane mirrors is further compressed to the Nd:YAG laser medium through the 6 fused silica aspherical lenses, evenly distributed around the common optical axis of both the laser rod and the spherical concentrator. The spherical concentrator has $40 \mathrm{~mm}$ radius and its center is positioned 18.5 $\mathrm{mm}$ above the aspherical lenses, which have an inclination angle of $7^{\circ}$ in relation to the z-axis for the efficient redirection of the pump light towards the laser rod, as shown in Fig. 3. These mounting positions and angles have been optimized to attain the best laser efficiency.

Both the aspherical lenses and the spherical concentrator are made of fused silica material, which is transparent over the Nd:YAG absorption spectrum. The high softening point of fused silica, its resistance to scratching and thermal shock, make this material very suitable for high solar flux pumping, preventing also the possible heating to the mechanical structure of the laser head. High optical quality fused silica lenses $(99.999 \%)$ can be manufactured by optical machining and polishing. The spherical concentrator can also be drilled for the installation of the Nd:YAG medium, the mechanical support and the cooling system. The back and lateral surfaces of the Nd:YAG laser medium are directly cooled by water, which also helps to eliminate some UV and IR radiation, that does not contribute to lasing. The maximum contact 
Return to the Manage Active Submissions page at http://spie.org/app/submissions/tasks.aspx and approve or disapprove this submission. Your manuscript will not be published without this approval. Please contact author_help@spie.org with any questions or concerns.

between the coolant and the active medium ensures the efficient removal of the generated heat. The cooled back surface of the Nd:YAG medium is $1064 \mathrm{~nm}$ HR coated (99.98\%), while front surface is $1064 \mathrm{~nm}$ AR coated. The laser resonator is formed by both the $1064 \mathrm{~nm}$ HR coating and the output coupler, as shown in Fig. 3. A hole is drilled along the mechanical support to allow the extraction of laser power.

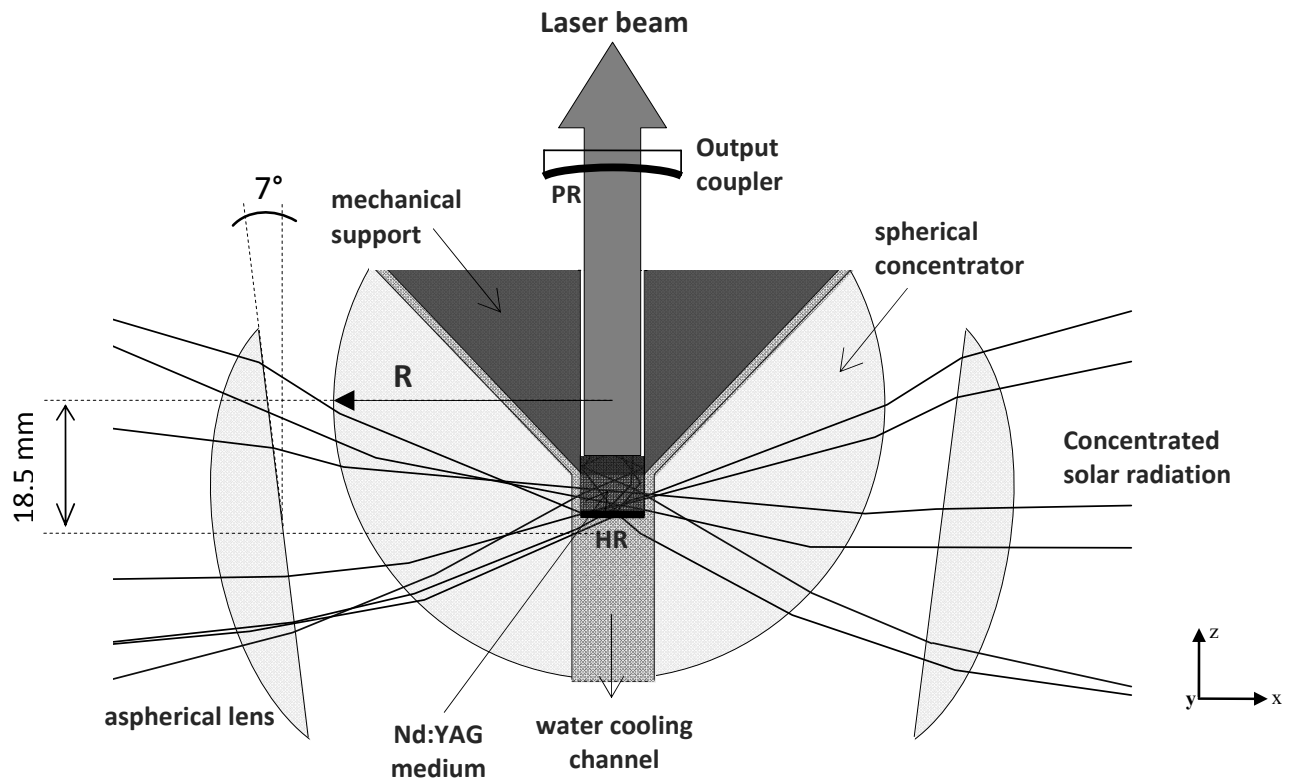

Fig. 3 - Cross-sectional side view of the pump light compression onto the Nd:YAG medium through the aspherical lenses and the spherical concentrator.

\section{NUMERICAL ANALYSIS OF THE Nd:YAG SOLAR LASER PERFORMANCE}

The optimal design of whole optical system is found through ZEMAX ${ }^{\mathrm{TM}}$ non-sequential ray-tracing. The standard solar spectrum for one-and-a-half air mass (AM1.5) [12] is used as the reference data for consulting the spectral irradiance $\left(\mathrm{W} / \mathrm{m}^{2} / \mathrm{nm}\right)$ at each wavelength. The terrestrial solar irradiance of $950 \mathrm{~W} / \mathrm{m}^{2}$ is considered in ZEMAX ${ }^{\mathrm{TM}}$ software. The effective pump power of the light source takes into account the $16 \%$ overlap between the absorption spectrum of the $\mathrm{Nd}$ :YAG medium and the solar spectrum [13]. The solar half-angle divergence of $0.27^{\circ}$ is also considered in the analysis. The absorption spectrum of PMMA, fused silica and water materials are included in ZEMAX $^{\mathrm{TM}}$ numerical data to account for absorption losses.

Despite the small overlap between the Nd:YAG absorption spectrum and the solar spectrum, Nd:YAG has been demonstrated as the best material under solar pumping because of its superior characteristic on thermal conductivity, high quantum efficiency and mechanical strength compared to other host materials [2-11]. For $1.1 \mathrm{at}^{\%} \mathrm{Nd}^{3+}$-doped YAG single-crystal medium, 22 absorption peaks are defined in $\mathrm{ZEMAX}^{\mathrm{TM}}$ numerical data. All the peak wavelengths and their respective absorption coefficients are added to the glass catalogue in $\mathrm{ZEMAX}^{\mathrm{TM}}$ software. Solar irradiance values for the above-mentioned 22 peak absorption wavelengths could be consulted from the standard solar spectra for AM1.5 and saved as source wavelength data. During ray-tracing, the active medium is divided into a total of 18000 zones. The path length in each zone is found. With this value and the effective absorption coefficient, the absorbed power within the laser medium can be calculated by summing up the absorbed pump radiation of all zones. The absorbed pump flux data from the ZEMAX ${ }^{\mathrm{TM}}$ analysis is then processed by LASCAD ${ }^{\mathrm{TM}}$ software to found the best laser beam parameters. 
Return to the Manage Active Submissions page at http://spie.org/app/submissions/tasks.aspx and approve or disapprove this submission. Your manuscript will not be published without this approval. Please contact author_help@spie.org with any questions or concerns.

In LASCAD ${ }^{\mathrm{TM}}$ analysis the optical resonator is comprised of two opposing parallel mirrors at right angles to the axis of the laser medium. The amount of feedback is determined by the reflectivity of the mirrors. One end mirror is high reflection coated, corresponding to the HR-coated surface of the active medium. The output coupler is partial reflection coated, with reflectivity variable between 90 - $99 \%$ according to different laser medium diameters. A $400 \mathrm{~mm}$ length resonator is chosen in $\mathrm{LASCAD}^{\mathrm{TM}}$ software. The adoption of the output coupler with $-5 \mathrm{~m}$ curvature radius leads to less laser beam divergence compared to that with smaller curvature radius. For the optimization of the laser system, the $\mathrm{Nd}$ :YAG laser output power is numerically study as function of the active medium diameter (D) and length (L). Maximum solar laser powers are numerically attained with $\mathrm{L}=9 \mathrm{~mm} \mathrm{Nd}$ :YAG single-crystal rods. Fig. 4 and Table 1 present the numerical solar laser results $P_{\text {LASER }}$, obtained with the $\mathrm{L}=9 \mathrm{~mm}$ rod of different diameters, and the correspondingly absorbed pump power $P_{A B S}$. The respective absorbed pump flux, $F_{A B S}$, is also given in Table 1 .

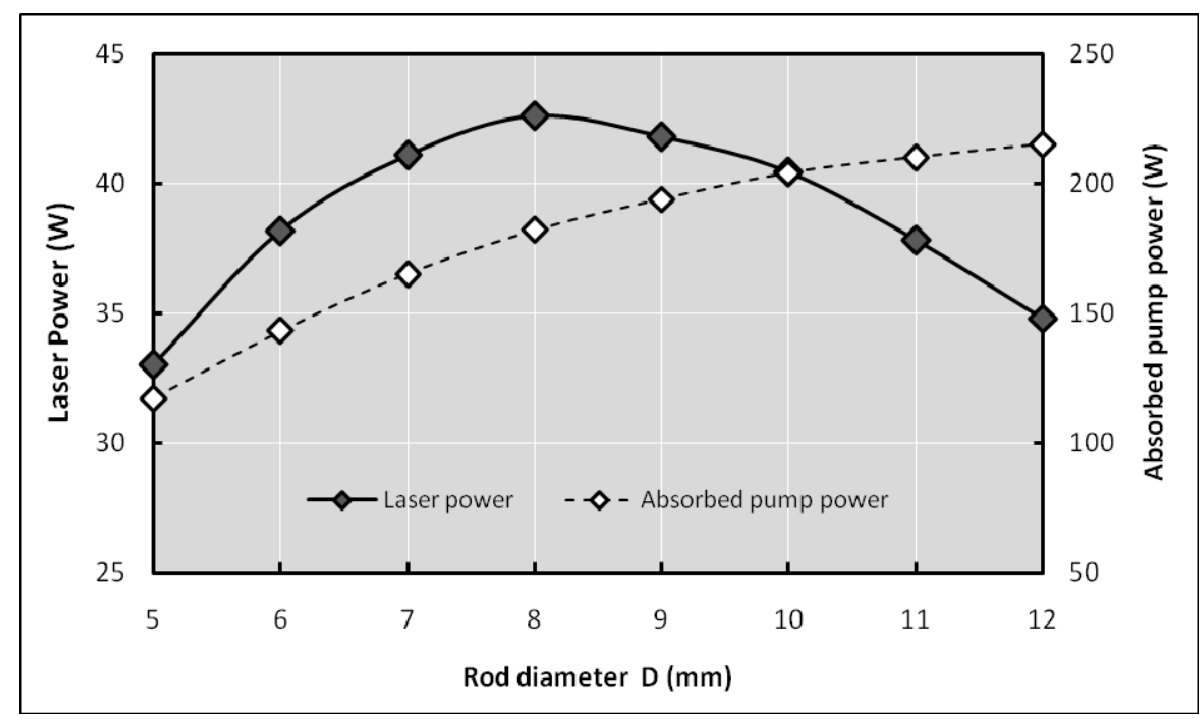

Fig 4. Numerically calculated Nd:YAG solar laser for different gain diameters (D) and respective absorbed pump power.

Table 1 - Numerical results of the absorbed pump power / flux and laser power as function of the Nd:YAG medium diameter

\begin{tabular}{ccccccccc}
\hline $\mathbf{D}(\mathbf{m m})$ & $\mathbf{5}$ & $\mathbf{6}$ & $\mathbf{7}$ & $\mathbf{8}$ & $\mathbf{9}$ & $\mathbf{1 0}$ & $\mathbf{1 1}$ & $\mathbf{1 2}$ \\
\hline $\mathbf{P}_{\text {ABS }}(\mathrm{W})$ & 117 & 143 & 165 & 182 & 194 & 204 & 210 & 215 \\
$\mathbf{F}_{\text {ABS }}\left(\mathrm{W} / \mathrm{mm}^{3}\right)$ & 0.66 & 0.56 & 0.48 & 0.40 & 0.34 & 0.29 & 0.25 & 0.21 \\
$\mathbf{P}_{\text {LASER }}(\mathrm{W})$ & 33.0 & 38.2 & 41.1 & 42.6 & 41.8 & 40.5 & 37.8 & 34.8 \\
\hline
\end{tabular}

Despite the increase of the absorbed pump power with larger diameter medium, the laser power follows a different tendency. Maximum multimode laser power of $42.6 \mathrm{~W}$ is numerically achieved with the $\mathrm{D}=8 \mathrm{~mm}$ by $\mathrm{L}=9 \mathrm{~mm}$ Nd:YAG single-crystal rod, from an absorbed pump power of $182 \mathrm{~W}$, as given in both Fig. 4 and Table 1. Significant collection efficiency of $18.5 \mathrm{~W} / \mathrm{m}^{2}$ is achieved. $118 \%$ more solar power can be absorbed by the $\mathrm{D}=12 \mathrm{~mm}$ by $\mathrm{L}=9 \mathrm{~mm}$ Nd:YAG rod. However, only $34.8 \mathrm{~W}$ laser power is produced in this case. These different behaviors could be explained by the significant reduction in absorbed pump flux, as observed in Table 1.

The grey-scale absorbed pump flux distribution within the central cross-sections of the $\mathrm{D}=8 \mathrm{~mm}$ by $\mathrm{L}=9 \mathrm{~mm} \mathrm{Nd}$ :YAG rod is shown in Fig. 5. Black color means near maximum pump absorption, whereas white means little or no absorption. The absorbed pump flux is slightly higher within the central region of the laser medium. $\mathrm{M}_{\mathrm{x}}{ }^{2}=\mathrm{M}_{\mathrm{y}}{ }^{2}=22$ factors are numerically found in this case, leading to the brightness figure of merit of $0.09 \mathrm{~W}$. This value is 14 times higher than that 


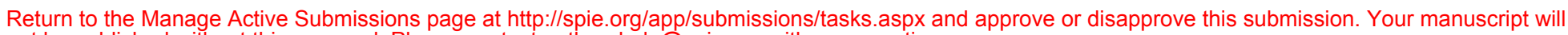
not be published without this approval. Please contact author_help@spie.org with any questions or concerns.

of the most powerful Nd:YAG solar laser [11]. By using a slightly complicated scheme with more Fresnel lenses and mirrors, a near uniform pump absorption profile can be obtained. This alleviates the thermal lensing effects [14] and the laser beam divergence is hence reduced. Thus, solar laser brightness figure of merit can be significantly improved.

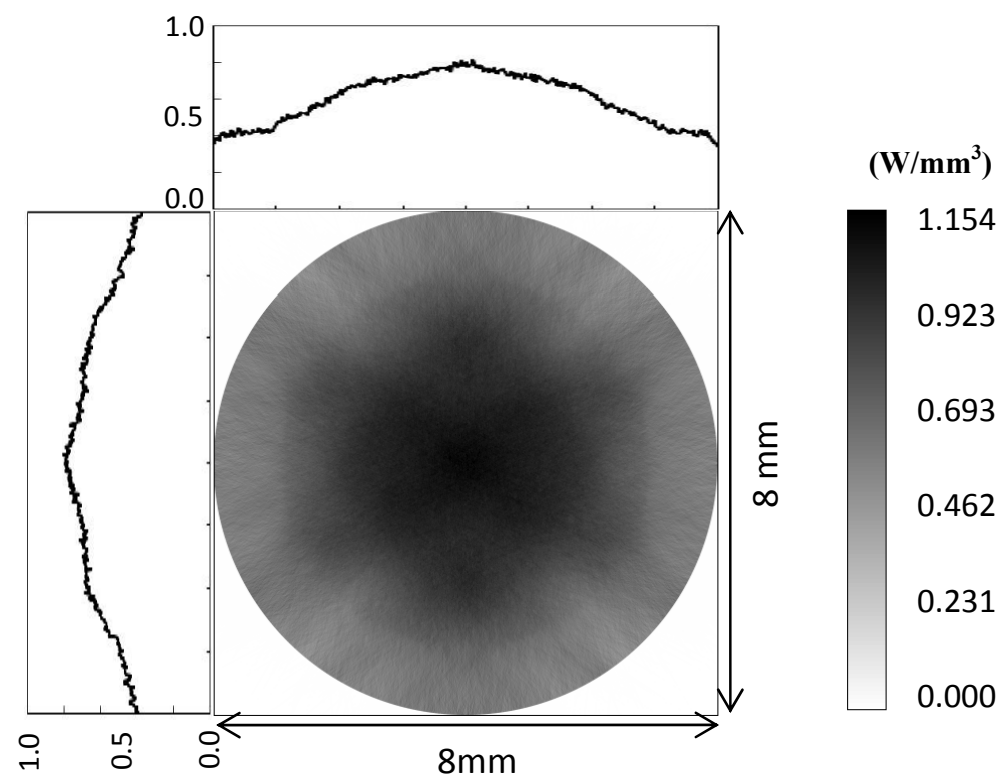

Fig 5. Absorbed pump flux distribution along the central cross-section of the $\mathrm{D}=8 \mathrm{~mm}$ by $\mathrm{L}=9 \mathrm{~mm} \mathrm{Nd}$ :YAG rod

\section{CONCLUSIONS}

A novel 6 Fresnel lenses - 6 plane mirrors solar laser pumping approach is put forward in this paper to achieve a good compromise between solar laser collection efficiency and brightness. About $1740 \mathrm{~W}$ solar power can be collected at the focal zone of this solar furnace scheme. By introducing the novel secondary concentrator, efficient side-pumping is applied to the Nd:YAG rod. $42.6 \mathrm{~W}$ solar laser power is numerically predicted for the $8 \mathrm{~mm}$ diameter by $9 \mathrm{~mm}$ length Nd:YAG single-crystal rod, corresponding to $18.5 \mathrm{~W} / \mathrm{m}^{2}$ collection efficiency. $\mathrm{M}_{\mathrm{x}}{ }^{2}=\mathrm{M}_{\mathrm{y}}{ }^{2}=22$ factors are numerically achieved. The brightness figure of merit of $0.09 \mathrm{~W}$ is hence deduced, surpassing by 14 times that of the most powerful Nd:YAG solar laser. A near uniform absorbed pump flux distribution can be attained by using more Fresnel lenses and mirrors. In our opinion, the proposed solar laser scheme can be an alternative for attaining both high collection efficiency and laser beam quality.

\section{ACKNOWLEDGEMENTS}

These research projects (PTDC/FIS/103599/2008 and PTDC/FIS/122420/2010) were funded by the Science and Technology Foundation of Portuguese Ministry of Science, Technology and Higher Education (FCT-MCTES).

\section{REFERENCES}

[1] Young, C. G., “A sun pumped cw one-watt laser,” Appl. Opt. 5(6), 993-997 (1966).

[2] Weksler, M. and Shwartz, J., "Solar-pumped solid-state lasers," IEEE J. Quantum Electron. 24(6), 12221228 (1988). 
Return to the Manage Active Submissions page at http://spie.org/app/submissions/tasks.aspx and approve or disapprove this submission. Your manuscript will not be published without this approval. Please contact author_help@spie.org with any questions or concerns.

[3] Arashi, H., Oka, Y., Sasahara, N., Kaimai, A. and Ishigame, M., “A solar-pumped cw 18 W Nd:YAG laser,” Jpn. J. Appl. Phys. 23 (8), 1051-1053 (1984).

[4] Benmair, R. M. J., Kagan, J., Kalisky, Y., Noter, Y., Oron, M., Shimony, Y. and Yogev, A., "Solar-pumped Er, Tm, Ho: YAG laser,” Opt. Lett. 15 (1), 36-38 (1990).

[5] Lando, M., Kagan, J., Linyekin, B. and Dobrusin, V., "A solar pumped Nd:YAG laser in the high collection efficiency regime," Opt. Commun. 222(1-6), 371-381 (2003).

[6] Almeida, J., Liang, D., Guillot, E. and Abdel-Hadi, Y., "A $40 \mathrm{~W}$ cw Nd:YAG solar laser pumped through a heliostat: a parabolic mirror system,” Laser Phys. 23(6), 065801-6 (2013).

[7] Yabe, T., Ohkubo, T., Uchida, S., Nakatsuka, M., Funatsu, T., Mabuti, A., Oyama, A., Nakagawa,Y., Oishi, T., Daito, K., Behgol, B., Nakayama,Y., Yoshida, M., Motokoshi, S., Sato, Y. and Baasandash, C., "High efficiency and economical solar energy pumped laser with Fresnel lens and chromium co-doped laser medium," Appl. Phys Lett. 90(26), 261120-3 (2007).

[8] Yabe, T., Bagheri, B., Ohkubo, T., Uchida, S., Yoshida, K., Funatsu, T., Oishi, T., Daito, K., Ishioka, M., Yasunaga, N., Sato, Y., Baasandash, C., Okamoto, Y., and Yanagitani, K., "100 W-class solar pumped laser for sustainable magnesium-hydrogen energy cycle,” J. Appl. Phys. 104(8), 083104 -1-8 (2008).

[9] Ohkubo, T., Yabe, T., Yoshida, K., Uchida, S., Funatsu, T., Bagheri, B., Oishi, T., Daito, K., Ishioka, M., Nakayama, Y., Yasunaga, N., Kido, K., Sato, Y., Baasandash, C., Kato, K., Yanagitani, T., and Okamoto, Y., "Solar-pumped 80 W laser irradiated by a Fresnel lens," Opt. Lett. 34(2), 175-177 (2009).

[10] Liang, D. and Almeida, J., "Highly efficient solar pumped Nd:YAG laser," Opt. Express 19(27), 26399-26405 (2011).

[11] Dinh, T. H., Ohkubo, T., Yabe, T. and Kuboyama, H., "120 watt continuous wave solar-pumped laser with a liquid light-guide lens and a Nd:YAG rod,” Opt. Lett. 37(13), 2670-2672 (2012).

[12] ASTM Standard G173 (2012).

[13] Zhao, B., Zhao, C., He, J. and Yang, S., "The Study of Active Medium for Solar-Pumped Solid-State Lasers," Acta Opt. Sin. 2007(10), 1-9 (2006).

[14] Brand, T., "Compact 170-W continuous-wave diode-pumped Nd:YAG rod laser with a cusp-shaped reflector, " Opt. Lett. 20(17), 1776-1778 (1995). 